\title{
Clinical utility of carotid and transcranial ultrasound in cerebrovascular diseases
}

This article was published in the following Dove Press journal:

Journal of Vascular Diagnostics

20 August 2014

Number of times this article has been viewed

\section{Lívia Figueiredo \\ Viviane $\mathrm{F}$ Zétola \\ Marcos C Lange}

Neurology Division, Hospital de Clínicas, Universidade Federal do Paraná, Curitiba, Brazil
Correspondence: Marcos C Lange Hospital de Clínicas, Serviço de Neurologia, Rua General Carneiro I8I, $4^{\circ}$ andar, 80060-900 Curitiba, Paraná, Brazil

Tel +55 4l 3252993 I

Fax +55 4I 32647500

Email langeneuro@gmail.com
Abstract: Carotid and transcranial (CTU) ultrasound is a useful tool in a number of clinical settings, particularly in cerebrovascular diseases. CTU is the only method that provides real-time determination of velocity and the spectral waveform of blood flow in the extracranial and basal intracranial arteries, and is effective in the detection of stenosis and occlusion. When transcranial ultrasound is considered, CTU is the only method that allows visualization of microembolic signals in the intracranial arteries. CTU makes a rapid differential diagnosis possible, improving therapeutic decision-making in acute stroke and determining the risk of recurrence and prognosis based on its findings. It is also the standard of care in children with sickle cell disease, when selecting patients for chronic blood transfusion, and for reducing the risk of ischemic stroke in these patients. CTU has some advantages, ie, relative simplicity in terms of interpretation and performance, and affordability, noninvasiveness, and portability. The main concern with ultrasound is that it is an operator-dependent tool and requires a high level of expertise and knowledge of three-dimensional cerebrovascular anatomy for correct interpretation of sonograms. The most significant limitation of intracranial evaluation by transcranial ultrasound is the absence of a suitable bone window in approximately $10 \%$ of patients. This paper gives an overview of the current utility and importance of CTU in the prevention and evaluation of ischemic cerebrovascular disease.

Keywords: transcranial Doppler ultrasonography, Doppler ultrasonography duplex, cerebrovascular disorders, stroke

\section{Introduction}

Carotid and transcranial ultrasound (CTU) is an important tool that can identify the etiology and differentiate the diagnosis in patients with cerebrovascular disease, offering additional findings such as the presence of microembolic signals during transcranial ultrasound (TCD) and identifying patients at high risk of recurrence when critical carotid or intracranial stenosis is observed. CTU has many advantages when compared with other vascular methods, in that it is noninvasive, operates in real time, and is portable.

The main concerns of CTU are its operator-dependent nature and the absence of a suitable bone window in approximately $10 \%$ of patients. ${ }^{1,2}$ Transcranial ultrasound also requires a high level of expertise and knowledge of three-dimensional cerebrovascular anatomy for correct interpretation of the sonograms. This paper gives an overview of the utility and importance of CTU in the prevention and evaluation of ischemic cerebrovascular disease.

\section{Sickle cell disease}

Sickle cell disease is a monogenetic hereditary disorder caused by abnormal hemoglobin, ie, hemoglobin S. As the disease progresses, the presence of hemoglobin S 
can lead to the development of renal failure, vaso-occlusive crisis, acute chest syndrome, and stroke. These complications reduce the life expectancy of patients with sickle cell disease by $25-30$ years when compared with the general population. ${ }^{3}$ Ischemic stroke secondary to occlusive intracranial vasculopathy is a serious complication in patients with sickle cell disease, and is the major cause of morbidity and mortality in children.,

The highest risk of ischemic stroke is in children aged 2-16 years. In one large study, the incidence of first ischemic stroke in patients with sickle cell disease was 0.08 events per 100 patients per year in children aged younger than 2 years; 0.75 events per 100 patients per year in patients aged 2-5 years; 0.55 events per 100 patients per year in children aged 6-9 years; and 0.30 events per 100 patients per year in children aged 10-19 years. ${ }^{4}$ Occlusive intracranial vasculopathy primarily involves the large vessels, ie, the internal carotid artery, middle cerebral artery, and anterior cerebral artery, and stenosis related to the vasculopathy may progress asymptomatically for many years. ${ }^{5}$

The authors of STOP (Stroke Prevention Trial in Sickle Cell Anemia) demonstrated that the use of chronic blood transfusions in children with sickle cell disease and increased mean cerebral blood flow velocity by TCD, suggesting severe stenosis of the intracranial basal arteries, had an approximately $90 \%$ reduction in relative risk of stroke compared with a control group. ${ }^{6}$ Subsequently, a primary prevention approach consisting of TCD monitoring and regular transfusion in patients with high TCD velocities has become standard of care in children with sickle cell disease. ${ }^{7}$ TCD should be performed and interpreted in accordance with the parameters defined by the STOP study, and should be done at least once a year in patients aged 2-16 years, based on previous guidelines. $^{8}$

Blood cell transfusion is indicated in patients with a mean cerebral blood flow velocity over $200 \mathrm{~cm}$ per second on two consecutive TCD examinations. The presence of mean cerebral blood flow velocity below $70 \mathrm{~cm}$ per second suggests severe stenosis and a repeat evaluation should be done 1 month later. ${ }^{8}$ It is important to note that chronic blood transfusion should not be interrupted by increasing age as suggested by the STOP-2 study, in which interruption of this procedure was associated with reversion to abnormal cerebral blood flow velocity and increased risk of stroke. ${ }^{9}$

\section{Spontaneous microembolic signals}

TCD is the only method that can be used to identify microembolic signals passing through the intracranial circulation.
Visualization of microembolic signals is possible due to the intensity of the abnormal substances (air, fat, or thrombus), which is higher than that of the blood flow inside the vessels. These abnormal signals are known as high intensity transitory signs and can be identified based on certain features, ie, high intensity, short duration, typical sound, unidirectional, and occurring in any moment of cardiac circle. ${ }^{10}$ Common sources of microembolic signals are the heart, the large arteries in the head and neck, and the venous system by a paradoxical embolism. In patients with ischemic stroke, transient ischemic attack, or asymptomatic high-grade internal carotid artery stenosis, searching for microembolic signals helps to establish the diagnosis and define the management strategy. ${ }^{11}$

Identification of microembolic signals contributes to improved diagnostic accuracy, supports therapeutic decisionmaking, and helps to predict the risk of cerebral ischemia and its recurrence. The presence of asymptomatic microembolic signals has been demonstrated to be an independent predictor of future stroke risk in patients with symptomatic or asymptomatic internal carotid artery stenosis. ${ }^{12-15}$ In some cases, the presence of microembolic signals may be the only finding in a patient with a proximal arterial dissection, a partially occlusive thrombus, or an unrecognized cardiac source of embolism. Embolic signals on TCD are also associated with intracranial clot dissolution. ${ }^{14-16}$

\section{Paradoxical embolism}

Abnormal right-to-left shunt, predominantly via patent foramen ovale, a congenital heart disease, is one of the most common causes of cryptogenic stroke in patients younger than 55 years of age. ${ }^{17}$ Transesophageal echocardiography (TEE) with contrast-enhanced technique (injection of saline solution in a peripheral vein) at rest and after the Valsalva maneuver is considered the gold standard for diagnosis of right-to-left shunt and patent foramen ovale. ${ }^{17-19}$ TEE visualizes the travel of a thrombus in real time and can provide anatomic details, such as an atrial septal aneurysm associated with patent foramen ovale. ${ }^{18}$ However, in addition to its advantages, TEE requires a high degree of expertise, is poorly tolerated by patients, and the Valsalva maneuver is often impaired by sedation. ${ }^{19}$

TCD has a high degree of accuracy for diagnosis of right-to-left shunt when compared with TEE. ${ }^{17-19}$ Further, TCD does not require sedation and is well tolerated by patients, and the Valsalva maneuver can be performed easily and reliably. ${ }^{18,19}$ A previous study that compared the ability of different methods to diagnose and quantify patent 
foramen ovale demonstrated that TCD with the Valsalva maneuver had higher sensitivity than TEE. ${ }^{17}$ When power M-mode Doppler is added, the sensitivity and specificity of TCD increases compared with TEE. ${ }^{20,21}$ Further, TCD can differentiate between an extracardiac right-to-left shunt and patent foramen ovale based on the number of microembolic signals and the latency time to the first microembolic signals after infusion of contrast. ${ }^{22}$

\section{Intracranial stenosis}

Intracranial atherosclerotic disease is increasingly recognized as a major cause of ischemic stroke and is an independent risk factor for recurrence of stroke, depending on sex and race. It accounts for approximately $8 \%$ of ischemic strokes. ${ }^{23-25}$ Patients with a previous transient ischemic attack or ischemic stroke secondary to intracranial stenosis have a $12 \%-14 \%$ risk of subsequent stroke in the 2 years after the event, despite treatment with antithrombotic medication. ${ }^{26}$

Diagnosis of intracranial stenosis is frequently done by digital subtraction angiography, which is considered to be the gold standard method but is high-risk and invasive. In this scenario, TCD emerges as a portable tool for more rapid screening and auxiliary diagnosis, with high sensitivity and specificity compared with digital subtraction angiography. For stenosis in the anterior intracranial circulation (internal carotid artery, middle cerebral artery, anterior cerebral artery), sensitivity is $70 \%-90 \%$ and specificity is $90 \%-95 \%{ }^{6,27}$ For stenosis in the posterior circulation, ie, intracranial vertebral arteries, basilar artery, and posterior cerebral arteries, the numbers are lower, ie, $50 \%-80 \%$ for sensitivity and $80 \%-96 \%$ for specificity. ${ }^{6}$

The SONIA (Stroke Outcomes and Neuroimaging of Intracranial Atherosclerosis) trial compared the accuracy of the different methods for detecting over 50\% intracranial stenosis. TCD had a positive predictive value of $57 \%-83 \%$ and a negative predictive value of $72 \%-86 \%$ when compared with digital subtraction angiography. Thus, SONIA demonstrated that TCD is a reliable tool to exclude the presence of intracranial stenosis. ${ }^{28}$ According to the SONIA criteria, mean cerebral blood flow velocity cutoffs were $100 \mathrm{~cm}$ per second for the middle cerebral artery and $80 \mathrm{~cm}$ per second for the intracranial vertebral arteries and basilar artery for identification of stenosis over $50 \%$ on TCD. More recent studies have shown better predictive values for TCD than those found in SONIA. ${ }^{29}$

In the SAMMPRIS (Stenting and Aggressive Medical Management for Preventing Recurrent Stroke in Intracranial Stenosis) trial, the authors validated TCD for stenosis greater than $70 \%$ compared with digital subtraction angiography. For the middle cerebral artery, the mean cerebral blood flow velocity over $120 \mathrm{~cm}$ per second and for the vertebral arteries and basilar artery, the mean cerebral blood flow velocity over $110 \mathrm{~cm}$ per second, are accepted as an indicator of $70 \%$ stenosis (SAMMPRIS criteria). ${ }^{30}$ Other important findings for intracranial stenosis are the presence of retrograde flow, turbulent flow, and musical murmurs at the site of stenosis and the stenotic/prestenotic flow velocity ratio. ${ }^{29,31-33}$

\section{Extracranial stenosis}

The pathogenesis of ischemic stroke secondary to extracranial internal carotid artery pathology is related to the presence of a significant stenosis. This stenosis is most commonly caused by an atherosclerotic plaque that reduces blood flow and has hemodynamic effects in the distal territory; in some situations, this plaque may generate emboli and occlusion of the distal arteries.

Carotid ultrasound has high reliability for classifying and grading stenosis, and takes morphology and complex hemodynamic parameters into account. These parameters represent physiological variables and could be related to the prognosis.

Grading of carotid stenosis by ultrasound should be based on hemodynamic and morphological information provided by B-mode, color flow, or B-flow imaging combined with Doppler sonography. The morphological study can classify the stenosis as low or moderate, based on area and reduction of diameter, as defined by NASCET (the North American Symptomatic Carotid Endarterectomy Trial). ${ }^{34}$ B-mode is one of the most reliable methods for demonstrating arterial wall thickness and minor plaques, providing images of the wall itself. However, in severe cases, cross-sectional B-mode images may be hindered by shadowing and other artifacts. The same is true for color flow. Therefore, the more severe the stenosis, the more important the hemodynamic criteria. ${ }^{35}$

In the assessment of hemodynamic effects, peak systolic velocity (PSV), measured by Doppler sonography, correlates with the narrowing measured in the stenotic segment. Many studies have examined the accuracy and reliability of this criterion for grading stenosis, and it has become accepted worldwide as a grading criterion. ${ }^{36-38}$

Based on hemodynamics, the velocity inside the vessel increases directly with increasing stenosis until a near occlusion, where the velocity reduces progressively. The correlation between velocity and degree of stenosis is demonstrated by Spencer's curve, a theoretical model of the relationship between flow velocity, flow volume, and decreasing size of 
the residual vessel lumen. ${ }^{39}$ In this regard, PSV as a single simplified diagnostic parameter for internal carotid artery stenosis is insufficient; it is recommended that the presence of collateral flow in the ophthalmic artery or anterior cerebral artery on TCD could be considered for severe stenosis. ${ }^{35}$

Finally, a multiparametric approach, with both morphological and hemodynamic criteria, might classify and grade carotid disease with high reliability into low-degree, moderate, or hemodynamically relevant stenosis. Low-degree stenosis $(0 \%-40 \%$, by NASCET criteria) is defined based on morphological information; moderate stenosis (50\%-60\% by NASCET criteria) combines locally increased velocity (PSV generally slower than $230 \mathrm{~cm} / \mathrm{sec}$ ), color flow, and B-mode imaging, generally with no collateral flow; and a hemodynamically relevant stenosis ( $>70 \%$ by NASCET criteria) is a combination of hemodynamic criteria (increased PSV or end-diastolic velocity) and presence of collateral flow. The post-stenotic flow velocity could be used in high-grade stenosis. The presence of a clear reduction of signal pulsatility and low post-stenotic velocity (PSV below $30 \mathrm{~cm} / \mathrm{sec}$ ), suggests a stenosis of approximately $90 \% .^{35}$

Another important evaluation in extracranial disease is the adequacy of cerebral perfusion status. ${ }^{40}$ Patient selection for intervention is generally based on the presence or absence of neurological symptoms and grading of the severity of carotid stenosis. TCD can further assist clinical decision-making because it helps to identify asymptomatic patients at potentially high risk of stroke by the absence of vasomotor reactivity (VMR) and the presence of intracranial embolization (microembolic signals). During intervention, TCD can be used to monitor intracerebral hemodynamics and brain embolization in real time, reducing perioperative complications. ${ }^{40}$

During carotid ultrasound, B-mode imaging allows evaluation of intima-media thickness, a surrogate end point for cardiovascular outcomes when evaluating the efficacy of modification of cardiovascular risk factors. ${ }^{41}$

\section{Cerebral vasomotor reactivity}

Cerebral VMR is defined as a change in cerebral blood flow velocity before and after administration of a vasodilatory stimuli (carbon dioxide inhalation reactivity test, intravenous acetazolamide injection and apnea) during TCD examination. ${ }^{42-44}$ All such stimuli produce an increase in the arterial carbon dioxide pressure, causing significant dilatation of the cerebral microvessels. The breath-holding test (apnea) needs active cooperation of the subject, who should hold his/her breath for a relatively long period of time (at least 30 seconds). ${ }^{44}$ The acetazolamide test, in which acetazolamide is administered in doses up to $1 \mathrm{~g}$, is a more potent cerebral vasodilator than induced hypercapnia (such as by apnea or carbon dioxide inhalation) and is useful for evaluating subtle abnormalities of cerebrovascular reserve, particularly in uncooperative patients. ${ }^{45}$

The utility of these VMR tests in evaluating intracranial hemodynamic status has been studied extensively in patients with internal carotid artery occlusive diseases, and has been shown to be a reliable marker for stroke risk in most but not all studies. ${ }^{46,47}$ Cerebral VMR tests could have a role in therapeutic decision-making for internal carotid artery disease, where the benefit-to-harm ratio for revascularization procedures is seemingly low. ${ }^{45-47}$ Patients with severe carotid stenosis or complete occlusion with abnormal VMR, in other words a smaller increase in mean middle cerebral artery blood flow velocity, are at high risk of stroke. ${ }^{45}$

During TCD evaluation, it is possible to identify the presence of collateral flow through the ophthalmic artery, anterior communicating cerebral artery, and posterior communicating cerebral artery. The potential for collateral blood flow in patients with carotid stenosis may influence management. For example, those who undergo carotid endarterectomy without evidence of collateral blood flow pathways on imaging may be at greater risk of a hypoperfusion infarct during temporary intraoperative carotid occlusion. Furthermore, patients with high-grade carotid stenosis or occlusion and low collateral blood flow to the distal middle cerebral artery territory may represent a group of patients in whom hemodynamic insufficiency plays a great role in causing transient or permanent neurologic deficits in the ipsilateral cerebral hemisphere. ${ }^{45}$

\section{Acute ischemic stroke}

TCD is considered effective in the detection of stenosis and occlusion of the basal cerebral arteries. The sensitivity and specificity of TCD are lower in comparison with other noninvasive imaging modalities, such as computed tomography angiography and magnetic resonance angiography. However, in terms of diagnosis of a lesion amenable to interventional treatment such as stenting, local thrombolysis, or angioplasty, TCD is a valuable tool for detection and monitoring of the effect of these interventions in acute ischemic stroke. ${ }^{15}$ The combination of TCD and carotid ultrasound in patients with acute ischemic stroke is a noninvasive portable method that allows the detection of poor prognosis findings and assists therapeutic 
decision-making with high accuracy when compared with digital subtraction angiography. ${ }^{31}$

\section{Treatment of acute stroke: thrombolysis}

CTU can be used successfully to determine vascular patency in patients with acute ischemic stroke because it can be done within minutes in the emergency room and even before admission to hospital. ${ }^{48}$ When performed by a trained person using a standardized and validated protocol, which should include both TCD and carotid ultrasound, this evaluation does not result in any delay in acute stroke management because it can be performed at the bedside simultaneously with neurological examination, monitoring of vital signs, and drawing blood. ${ }^{15,31}$

CTU in patients with acute ischemic stroke helps to detect major intracranial arterial occlusion and carotid stenosis, both of which are associated with poor neurological recovery. Information about collateral pathways and the presence of microembolic signals provided by TCD helps to ascertain the pathogenic mechanism of stroke.

TCD can also be used to monitor the efficacy of treatment with intravenous recombinant tissue plasminogen activator (rtPA) for acute ischemic stroke, and is the only tool that can monitor thrombolysis-induced reperfusion in real time. The timing of brain reperfusion detected by TCD is closely associated with dramatic clinical recovery during rtPA infusion. However, one third of early complete recanalization procedures do not result in immediate clinical improvement, despite being detected by TCD. ${ }^{49}$ One third of those patients have a complete clinical recovery at 3 months. ${ }^{15}$

One of the most common classifications used to monitor changes during treatment with rtPA is TIBI (Thrombolysis in Brain Ischemia). The TIBI grade correlates with initial stroke severity, mortality, likelihood of reperfusion, and clinical improvement. ${ }^{50}$ In the multicenter NAIS (Neurosonology in Acute Ischemic Stroke) study, the sonographic status of the middle cerebral artery within the 6 hours after stroke was found to be an independent predictor of outcome. ${ }^{51}$

CTU can rapidly identify patients with acute arterial occlusion amenable to an interventional approach, regardless of the baseline stroke severity. Key ultrasound findings for diagnosis of a lesion amenable to intervention include: one of four abnormal TIBI waveforms (absent, minimal, blunted, dampened) in the vessel supplying a territory affected by ischemia; and evidence of flow diversion or collateralization to compensate for this lesion. ${ }^{52}$
The degree of recanalization can be detected by TCD spectral configuration changes. ${ }^{53} \mathrm{~A}$ rapid increase in TIBI grade indicates rapid and more complete clot lysis, and high diastolic velocity indicates low resistance in the distal circulatory bed. In contrast, slow TIBI improvement and dampened waveform are ultrasonic signs of incomplete recanalization. Persistence of a low TIBI grade after intravenous thrombolysis can indicate a need for further reperfusion therapies, such as intra-arterial thrombolysis or mechanical thrombectomy. ${ }^{54}$ In addition, an abundance of microembolic signals on TCD examination can indicate a need for early initiation of anticoagulation or antiplatelet therapy. On the other hand, a hyperemic flow pattern can help the clinician to modify blood pressure limits. ${ }^{55}$

\section{Sonothrombolysis}

Intravenous rtPA infusion is the fastest way to initiate thrombolytic therapy; however, poor recovery might be expected in up to $50 \%$ of patients, as a result of the initial severity of the ischemic insult and slow and incomplete thrombolysis. When given intravenously, delivery of rtPA to the thrombus is dependent on the residual flow to and around the arterial obstruction, and better residual flow signals are associated with higher recanalization rates in stroke patients treated with rtPA. A $2 \mathrm{mHz}$ pulsed-wave diagnostic ultrasound beam can serve several purposes: to identify the presence of obstructive intracranial thrombus in a vessel; to provide real-time bedside monitoring of thrombolysis; and to augment residual flow and speed up thrombolysis, allowing patients to recover from stroke more rapidly and completely. ${ }^{52}$ Therefore, therapeutic ultrasound is a promising stroke treatment paradigm.

The ultrasound exposure enhances both spontaneous (sonothrombolysis) and thrombolytic agent-mediated lysis of an intravascular clot (ultrasound-enhanced thrombolysis). The mechanisms include facilitation of permeation of the thrombolytic agent into the clot by promoting fluid motion around the clot surface (microstreaming), formation of micropores or opening of the cross-linked fibrin mesh, direct cleavage of fibrin polymers, and the creation of extra binding sites for rtPA on the fibrin network. ${ }^{52,56,57}$

CLOTBUST (Combined Lysis of Thrombus in Brain Ischemia Using Transcranial Ultrasound and Systemic t-PA) was a randomized, multicenter clinical trial that demonstrated a $49 \%$ rate of complete recanalization within 2 hours after a bolus of rtPA when rtPA infusion was continuously monitored with TCD, compared with a $30 \%$ rate of complete recanalization in patients who received rtPA without ultrasound monitoring, with no increase of intracranial 
hemorrhage. ${ }^{56}$ This increased recanalization rate translated into an increased rate of clinical recovery after 3 months, with $41.5 \%$ of patients in the monitored TCD group achieving a favorable outcome compared with $28 \%$ in the control group. ${ }^{56}$

However, delivery of sonothrombolysis via cranial bone windows requires training in both anatomic localization and waveform recognition. The development of an operatorindependent device that can target the proximal intracranial arteries without specialized neurovascular ultrasound training would make the sonothrombolysis technique easier. A device was developed and has now undergone preliminary (Phase I) testing. ${ }^{57}$ This hands-free device uses $2 \mathrm{mHz}$ pulsed-wave technology that never exceeds approved diagnostic ultrasound exposure levels and delivers energy through both temporal windows and the suboccipital window via 18 separate transducers. The CLOTBUST-HF (Combined Lysis of Thrombus in Brain Ischemia With Transcranial Ultrasound and Systemic T-PA-Hands-Free) study tested the safety of the handsfree device in a cohort of patients with ischemic stroke. ${ }^{57}$ Compared with historical controls (who received rtPA alone) from the original CLOTBUST study, ${ }^{56}$ patients treated with the hands-free device experienced higher rates of sustained complete recanalization, similar to those observed with the hand-held device. All patients tolerated the entire 2 hours of insonation, and none developed symptomatic intracerebral hemorrhage. ${ }^{57}$

\section{Conclusion}

CTU, a combination of TCD and carotid ultrasound, has an important role in the rapid diagnosis of carotid and intracranial diseases, in improving therapeutic decisionmaking in acute stroke, in assessing the risk of recurrence and poor prognosis, and for primary prevention in asymptomatic patients. Technological advances in these methods can lead to their greater relevance in the context of neurological diseases. CTU, as rapid and informative emergency vascular assessment tool, has the advantage of being a portable, noninvasive, and affordable technique.

TCD is the only noninvasive real-time neuroimaging modality for evaluation of the characteristics of cerebral blood flow velocity in the basal intracranial vessels that adds physiological information to structural imaging. TCD has become an important tool for diagnosis and monitoring cerebrovascular pathologies. TCD has become an important tool for diagnosis and monitoring cerebrovascular pathologies.

Within its large spectrum of clinical applications, TCD is a standard of care in children with sickle cell disease, reducing the risk of stroke in these patients. In acute stroke, TCD provides rapid information about vascular stenosis and occlusion and the hemodynamic status of the cerebral circulation, and allows real-time monitoring of recanalization. Extended applications such as VMR, monitoring of microembolic signals, and detection of right-to-left shunt help clinicians to ascertain stroke mechanisms at the bedside, plan and monitor treatment, and determine prognosis.

\section{Disclosure}

The authors report no conflicts of interest in this work.

\section{References}

1. Zetola VF, Lange MC, Muzzio JA, Marchioro I, Nóvak EM, Werneck LC. Doppler transcraniano na prática neurológica. [Transcranial Doppler in the neurological practice]. Arq Neuropsiquiatr. 2006;64(1):100-103. Portuguese.

2. Suri MF, Georgiadis AL, Tariq N, Vazquez G, Qureshi N, Qureshi AI. Estimated prevalence of acoustic cranial windows and intracranial stenosis in the US elderly population: ultrasound screening in adults for intracranial disease study. Neuroepidemiology. 2011;37(1):64-71.

3. Platt OS, Brambilla DJ, Rosse WF, et al. Mortality in sickle cell disease. Life expectancy and risk factors for early death. $N$ Engl J Med. 1994;330(23):1639-1644.

4. Ohene-Frempong K, Weiner SJ, Sleeper LA, et al. Cerebrovascular accidents in sickle cell disease: rates and risk factors. Blood. 1998;91(1): 288-294.

5. Bulas D. Screening children for sickle cell vasculopathy: guidelines for transcranial Doppler evaluation. Pediatr Radiol. 2005;35(3): 235-241.

6. Adams RJ, McKie VC, Brambilla D, et al. Stroke prevention trial in sickle cell anemia. Control Clin Trials. 1998;19(1):110-129.

7. Sloan MA, Alexandrov AV, Tegeler CH, et al. Assessment: transcranial Doppler ultrasonography: report of the Therapeutics and Technology Assessment Subcommittee of the American Academy of Neurology. Neurology. 2004;62(9):1468-1481.

8. Lobo CLC, Cançado RD, Leite AC, et al. Brazilian guidelines for transcranial Doppler in children and adolescents with sickle cell disease. Rev Bras Hematol Hemoter. 2011;33(1):43-48.

9. Adams RJ, Brambilla D; Optimizing Primary Stroke Prevention in Sickle Cell Anemia (STOP 2) Trial Investigators. Discontinuing prophylactic transfusions used to prevent stroke in sickle cell disease. N Engl J Med. 2005;353(26):2769-2778.

10. Ringelstein EB, Drost DW, Babikian VL, et al. Consensus on microembolus detection by TCD. International Consensus Group on Microembolus Detection. Stroke. 1998;29(3):725-729.

11. Alexandrov AV, Sloan MA, Tegeler CH, et al. Practice standards for transcranial Doppler (TCD) ultrasound. Part II. Clinical indications and expected outcomes. J Neuroimaging. 2012;22(3):215-224.

12. Markus HS, King A, Shipley M, et al. Asymptomatic embolisation for prediction of stroke in the Asymptomatic Carotid Emboli Study (ACES): a prospective observational study. Lancet Neurol. 2010;9(7):663-671.

13. King A, Markus HS. Doppler embolic signals in cerebrovascular disease and prediction of stroke risk: a systematic review and meta-analysis. Stroke. 2009;40(12):3711-3717.

14. Molloy J, Markus HS. Asymptomatic embolization predicts stroke and TIA risk in patients with carotid artery stenosis. Stroke. 1999;30(7):1440-1443.

15. Kalanuria A. Use of transcranial Doppler ultrassound in the neurocritical care unit. Neurosurg Clin N Am. 2013;24(3):441-456.

16. Alexandrov AV, Demchuk AM, Felberg RA, Grotta JC, Krieger DW. Intracranial clot dissolution is associated with embolic signals on transcranial Doppler. J Neuroimaging. 2000;10(1):27-32. 
17. Gonzãlez-Alujas T, Evangelista A, Santamarina E, et al. Diagnosis and quantification of patent foramen ovale. Which is the reference technique? Simultaneous study with transcranial Doppler, transthoracic and transesophageal echocardiography. Rev Esp Cardiol. 2011;64(2): 133-139.

18. Lange MC, Zetola VF, de Souza AM, et al. Transcranial Doppler for patent foramen ovale screening. Is there a good correlation with transesophageal echocardiography? Arq Neuropsiquiatr. 2008;66(4):785-789.

19. Jauss M, Kaps M, Keberle M, Haberbosch W, Dorndorf W. A comparison of transesophageal echocardiography and transcranial Doppler sonography with contrast medium in the detection of patent foramen ovale. Stroke. 1994;25(6):1265-1267.

20. Spencer MP, Moehring MA, Jesurum J, Gray WA, Olsen JV, Reisman M. Power M-mode transcranial Doppler for diagnosis of patent foramen ovale and assessing transcatheter closure. J Neuroimaging. 2004;14(4) 342-349.

21. Saqqur M, Dean N, Schebel M, et al. Improved detection of microbubble signals using power M-mode Doppler. Stroke. 2004;35(1): e14-e17.

22. Lange MC,ZetolaVF, De SouzaAM, NóvakFM, Piovesan EJ, Werneck LC Intracranial embolism characteristics in PFO patients: a comparison between positive and negative PFO by transesophageal echocardiography: the rule of nine. J Neurol Sci. 2010;293(1-2):106-109.

23. White $H$, Boden-Albala $B$, Wang $C$, et al. Ischemic stroke subtype incidence among whites, blacks, and Hispanics: the North Manhattan Study. Circulation. 2005;111(10):1327-1331.

24. Sacco RL, Kargman DE, Gu Q, Zamanillo MC. Rate-ethnicity and determinants of intracranial atherosclerotic cerebral infarction. Stroke. 1995;26(1):14-20.

25. Wityk RJ, Lehman D, Klag M, Coresh J, Ahn H, Litt B. Race and sex differences in the distribution of cerebral atherosclerosis. Stroke. 1996;27(11):1974-1980.

26. Mazighi M, Tanasescu R, Ducrocq X, et al. Prospective study of symptomatic atherothrombotic intracranial stenoses: the GESICA study. Neurology. 2006;66(8):1187-1191.

27. Felberg RA, Christou I, Demchuk AM, Malkoff M, Alexandrov AV. Screening for intracranial stenosis with transcranial Doppler: the accuracy of mean flow velocity tresholds. J Neuroimaging. 2002;12(1) 9-14

28. Feldmann E, Wilterdink JL, Kosinski A, et al. The Stroke Outcomes and Neuroimaging of Intracranial Atherosclerosis (SONIA) trial. Neurology. 2001;68(24):2099-2106.

29. Zhao L, Barlinn K, Sharma VK, et al. Velocity criteria for intracranial stenosis revisited. An international multicenter study of transcrania doppler and digital subtraction angiography. Stroke. 2011;42(12): 3429-3434.

30. Chimowitz MI, Lynn MJ, Derdeyn CP, et al. Stenting versus aggressive medical therapy for intracranial arterial stenosis. $N$ Engl J Med. 2011;365(11):993-1003.

31. Chernyshev OY, Garami Z, Calleja S, et al. Yield and accuracy of urgent combined carotid/transcranial ultrasound testing in acute cerebral ischemia. Stroke. 2005;36(1):32-37.

32. Nedelmann M, Stolz E, Gerriets T, et al; TCCS Consensus Group. Consensus recommendations for transcranial color-coded duplex sonography for the assessment of intracranial arteries in clinical trials on acute stroke. Stroke. 2009;40(10):3238-3244.

33. Baumgartner RW. Intracranial stenoses and occlusions, and circle of Willis collaterals. In: RW Baumgartner, editor. Handbook on Neurovascular Ultrasound. Basel, Switzerland: Karger; 2006.

34. North American Symptomatic Carotid Endarterectomy Trial (NASCET) Collaborators. Beneficial effect of carotid endarterectomy in symptomatic patients with high-grade carotid stenosis. N Engl J Med. 1991; 325(7):445-453.

35. von Reutern GM, Goethler MW, Bornstein NM, et al. Grading carotid stenosis using ultrasonic methods. Stroke. 2012;43(3):916-921.

36. Alexandrov AV, Bladin CF, Maggisano R, Norris JW. Measuring carotid stenosis. Time for a reappraisal. Stroke. 1993;24(9):1292-1296.
37. de Bray JM, Glatt B. Quantification of atheromatous stenosis in the extracranial internal carotid artery. Cerebrovasc Dis. 1995;5(6): 414-426.

38. Grant EG, Benson CB, Moneta GL, et al. Carotid artery stenosis: grayscale and Doppler US diagnosis - Society of Radiologists in Ultrasound Consensus Conference. Radiology. 2003;229(2):340-346.

39. Alexandrov AV. The Spencer's curve: clinical implications of a classic hemodynamic model. J Neuroimaging. 2007;17(1):6-10.

40. Alexandrov AV, Babikian VL, Adams RJ, et al. The evolving role of transcranial Doppler in stroke prevention and treatment. J Stroke Cerebrovasc Dis. 1998;7(2):101-104.

41. Touboul PJ, Hennerici MG, Meairs S, et al. Mannheim carotid intimamedia thickness and plaque consensus (2004-2006-2011). An update on behalf of the advisory board of the $3 \mathrm{rd}$, 4th and 5 th watching the risk symposia, at the 13th, 15th and 20th European Stroke Conferences, Mannheim, Germany, 2004, Brussels, Belgium, 2006, and Hamburg, Germany, 2011. Cerebrovasc Dis. 2012;34(4):290-296.

42. Ringelstein EB, Sievers C, Ecker S, Schneider PA, Otis SM. Noninvasive assessment of $\mathrm{CO}_{2}$-induced cerebral vasomotor response in normal individuals and patients with internal carotid artery occlusions. Stroke. 1998;19(8):963-969.

43. Dahl A, Russell D, Rootwelt K, Nyberg-Hansen R, Kerty E. Cerebral vasoreactivity assessed with transcranial Doppler and regional cerebral blood flow measurements. Dose, serum concentration, and time course of the response to acetazolamide. Stroke. 1995;26(12):2302-2306.

44. Markus HS, Harrison MJ. Estimation of cerebrovascular reactivity using transcranial Doppler, including the use of breath-holding as the vasodilatory stimulus. Stroke. 1992;23(5):668-673.

45. Russel SM, Woo HH, Siller K, Panasci D, Leroux PD. Evaluating middle cerebral artery collateral blood flow reserve using acetazolamide transcranial Doppler ultrasound in patients with carotid occlusive disease. Surg Neurol. 2008;70(5):466-470.

46. Silvestrini M, Vernieri F, Pasqualetti $P$, et al. Impaired cerebral vasoreactivity and risk of stroke in patients with asymptomatic carotid artery stenosis. JAMA. 2000;283(16):2122-2127.

47. King A, Serena J, Bornstein NM, Markus HS. Does impaired cerebrovascular reactivity predict stroke risk in asymptomatic carotid stenosis? A prospective substudy of the asymptomatic carotid emboli study. Stroke. 2011;42(6):1550-1555.

48. Herzberg M, Boy S, Hölscher T, et al. Prehospital stroke diagnostics based on neurological examination and transcranial ultrasound. Crit Ultrasound J. 2014;6(1):3.

49. Alexandrov AV, Hall CE, Labiche LA, Wojner AW, Grotto JC. Ischemic stunning of the brain: early recanalization without immediate clinical improvement in acute ischemic stroke. Stroke. 2004;35(2):449-452.

50. Demchuk AM, Burgin WS, Christou I, et al. Thrombolysis in Brain Ischemia (TIBI) transcranial Doppler flow grades predict clinical severity, early recovery, and mortality in patients treated with intravenous tissue plasminogen activator. Stroke. 2001;32(1):89-93.

51. Goertler M, Allendoefer J, von Reutern GM. The Neurosonology in Acute Ischaemic Stroke (NAIS) Study Group. Design of a multicentre study on neurosonology in acute ischaemic stroke: A Project of the Neurosonology Research Group of the World Federation of Neurology. Eur J Ultrasound. 2002;16(1-2):115-120.

52. Alexandrov AV. Ultrasound identification and lysis of clots. Stroke. 2004;35(11 Suppl 1):2722-2725.

53. Alexandrov AV, Burgin WS, Demchuk AM, El-Mitwalli A, Grotta JC. Speed of intracranial clot lysis with intravenous tissue plasminogen activator therapy: sonographic classification and short-term improvement. Circulation. 2001;103(24):2897-2902.

54. Saqqur M, Shuaib A, Alexandrov AV, et al. Derivation of transcranial Doppler criteria for rescue intra-arterial thrombolysis: multicenter experience from the Interventional Management of Stroke study. Stroke. 2005;36(4):865-868.

55. Burgin WS, Malkoff M, Felberg RA, et al. Transcranial Doppler ultrasound criteria for recanalization after thrombolysis for middle cerebral artery stroke. Stroke. 2000;31(5):1128-1132. 
56. Alexandrov AV, Molina CA, Grotta JC, et al. Ultrasound-enhanced systemic thrombolysis for acute ischemic stroke. $N$ Engl J Med. 2004;351(21):2170-2178.
57. Barlinn K, Barreto AD, Sisson A, et al. CLOTBUST-hands free: initial safety testing of a novel operator-independent ultrasound device in stroke-free volunteers. Stroke. 2013;44(6):1641-1646.

\section{Publish your work in this journal}

Journal of Vascular Diagnostics is an international, peer-reviewed journal of diagnostics, focusing on non invasive vascular investigation methods involved in the evaluation of vascular diseases. The journal is committed to the rapid publication in the fields of vascular diseases. Original research, review, case reports, expert opinion and commentaries are all considered for publication. The manuscript management system is completely online and includes a very quick and fair peer-review system, which is all easy to use. Visit http://www.dovepress. com/testimonials.php to read real quotes from published authors. 